\title{
Désensibilisation de la photolyse du formiate d'uranyle
}

\section{Doctoral Thesis}

Author(s):

Ouellet, Cyrias

Publication date:

1931

Permanent link:

https://doi.org/10.3929/ethz-a-000095050

Rights / license:

In Copyright - Non-Commercial Use Permitted

Originally published in:

Sammelband ETH-BIB 


\section{DÉSENSIBILISATION DE LA PHO TOLYSE DU FORMIATE D'URANYLE}

\section{TH È SE}

présenté à l'Ecole Polytechnique Fédérale, Zurich, pour l'obtention du grade de Docteur ès Sciences tedniques

$$
\begin{gathered}
\text { par } \\
\text { Cyrias Ouellet } \\
\text { de Québec, Canada }
\end{gathered}
$$

Nr. 665

$$
\begin{aligned}
& \text { Rapporteur: } \text { M. le professeur } D^{r} \text { E. Baur } \\
& \text { Corapporteur: } \\
& \text { M. le professeur } D^{r} \text { W. D. Treadwell }
\end{aligned}
$$

B A L E

Imprimerie Emile Birkhæuser \& Cie.

$$
193_{1}
$$


L'équation (11) avait été déduite par $B a u r^{1}$ ) en introduisant, pour la désensibilisation par $\mathrm{I}_{2}$, la formule (7) qui n'a pas été vérifiée. Voyons à quel résultat on arrive en exprimant la désensibilisation par la formule (13). Nous avons done la photolyse

$$
\mathrm{U}^{\mathrm{VI}}\left\{\begin{array}{l}
\oplus \oplus+2 \mathrm{I}^{\prime} \longrightarrow \mathrm{I}_{2} \\
\ominus \ominus+\mathrm{U}^{\mathrm{VI}} \longrightarrow \mathrm{U}^{\mathrm{IV}}
\end{array}\right.
$$

et la réaction thermique inverse

$$
\mathrm{I}_{2}+\mathrm{U}^{\mathrm{IV}} \longrightarrow 2 \mathrm{I}^{\prime}+\mathrm{U}^{\mathrm{VI}}
$$

La vitesse de (8) s'écrit, en tenant compte de l'auto-désensibilisation par $\mathrm{I}_{2}$ :

$$
\frac{d\left(\mathrm{I}_{2}\right)}{d t}=\frac{\lambda L}{a+b\left(\mathrm{I}_{2}\right)}=\lambda L\left(1-\frac{\alpha\left(\mathrm{I}_{2}\right)}{1+\beta\left(\mathrm{I}_{2}\right)}\right)
$$

en vertu de l'équivalence de (6) et (13), démontrée plus haut ( $a$, • $b, \alpha, \beta$ et $\lambda$ sont des constantes).

D'autre part, la vitesse de (9) sera

$$
-\frac{d\left(\mathrm{I}_{2}\right)}{d t}=\mathrm{K} \cdot\left(\mathrm{U}^{\mathrm{IV}}\right)\left(\mathrm{I}_{2}\right)=\mathrm{K}\left(\mathrm{I}_{\mathrm{i}}\right)^{2}
$$

puisque, d'après (8), $\mathrm{U}^{\mathrm{IV}}$ et $\mathrm{I}_{2}$ sont en quantités stoechiométriquement équivalentes. A l'équilibre, les vitesses des réactions (8) et (9) s'égalent, c'est-à-dire

ou bien

$$
\mathrm{K}\left(\mathrm{I}_{2}\right)^{2}=\lambda L-\frac{\lambda L \cdot \alpha\left(\mathrm{I}_{2}\right)}{1+\beta\left(\mathrm{I}_{2}\right)}
$$

$$
\begin{aligned}
\mathrm{K}\left(\mathrm{I}_{2}\right) & =\frac{\lambda L}{\left(\mathrm{I}_{2}\right)}-\frac{\alpha \cdot \lambda L}{1+\beta\left(\mathrm{I}_{2}\right)} \\
\frac{\lambda L}{\left(\mathrm{I}_{2}\right)} & =\mathrm{K}\left(\mathrm{I}_{2}\right)+\frac{\alpha \cdot \lambda L}{1+\beta\left(\mathrm{I}_{2}\right)}=\frac{\mathrm{K}\left(\mathrm{I}_{2}\right)\left[1+\beta\left(\mathrm{I}_{2}\right)\right]+\alpha \cdot \lambda L}{1+\beta\left(\mathrm{I}_{2}\right)} \\
\left(\mathrm{I}_{2}\right) & =\frac{\left[1+\beta\left(\mathrm{I}_{2}\right)\right] \lambda L}{\mathrm{~K}\left(\mathrm{I}_{2}\right)\left[1+\beta\left(\mathrm{I}_{2}\right)\right]+\alpha \lambda L}
\end{aligned}
$$

Comme $\left(\mathrm{I}_{2}\right)$ est petit et croît lentement, $(25)$ peut s'écrire, en première approximation,

$$
\left(\mathrm{I}_{2}\right)_{s}=\frac{L}{a+b L}
$$

$a$ et $b$ étant des constantes. On retrouve bien la même formule.

\section{Résumé et conclusions.}

Des recherches ont été faites sur la désensibilisation de la photolyse du formiate d'uranyle, pour trouver une relation entre la vitesse de la photolyse et la concentration du désensibilisateur. Les résultats obtenus permettent de tirer les conclusions suivantes:

1) E. Baur, Helv. 12, 796 (1929). 
1. La vitesse de la photolyse dépend des concentrations de l'acide formique et de l'ion uranyle.

2. La photolyse est désensibilisée puissamment par I', $\mathrm{Cl}^{\prime}, \mathrm{Fe} \cdot$, l'hydroquinone, $\mathrm{K}_{2} \mathrm{Cr}_{2} \mathrm{O}_{7}, \mathrm{AgNO}_{3}, \mathrm{NaNO}_{2}, \mathrm{KCN}, \mathrm{HgSO}_{4}$, faiblement par $\mathrm{CuSO}_{4}, \mathrm{Cr}_{2}\left(\mathrm{SO}_{4}\right)_{3}, \mathrm{NaNO}_{3}, \mathrm{KF}$, tandis que $\mathrm{Na}_{3} \mathrm{PO}_{4}, \mathrm{NH}_{4} \mathrm{COO} \cdot \mathrm{CH}_{3}$ et $\mathrm{MgSO}_{4}$ sont sans influence. Les propriétés oxydo-réductrices des désensibilisateurs jouent un rôle important.

3. Quand on augmente la concentration du désensibilisateur, l'effet croît d'abord rapidement, puis de plus en plus lentement, de sorte que les premières traces sont relativement les plus actives. La vitesse $v$ de la photolyse désensibilisée est donnée, en fonction de la vitesse $v_{0}$ de la photolyse non désensibilisée et de la concentration $(D)$ $\mathrm{du}$ désensibilisateur, par la relation

$$
v=\frac{v_{0}}{\alpha+\beta(D)},
$$

où $\alpha$ et $\beta$ sont des constantes. Cette équation est de même forme que celle qui a été proposée par Baur pour le cas de l'auto-désensibilisation et par Bäckström pour l'action anti-oxygène, et discutée théoriquement par Christiansen.

4. Il est donné de cette formule une déduction théorique générale. Le raisonnement suivi laisse prévoir que l'effet du désensibilisateur doit être d'autant plus grand que la concentration du photolyte est plus faible, ce que l'expérience a vérifié.

5. Dans le cas de la photolyse réversible de KI en présence de $\mathrm{UO}_{2} \mathrm{SO}_{4}$, la concentration de $\mathrm{I}_{2}$ stationnaire augmente de moins en moins avec l'intensité lumineuse, suivant la formule proposée par Baur

$$
\left(\mathbf{I}_{2}\right)_{s}=\frac{L}{a+b L},
$$

où $L$ est l'éclairement, tandis que $a$ et $b$ sont des constantes.

Ces recherches ont été proposées par M. le professeur Emil Baur et effectuées sous sa direction au laboratoire de Chimie-physique de l'Ecole polytechnique fédérale à Zurich. Je tiens à remercier ici M. le professeur Baur de ses conseils éclairés et de l'intérêt bienveillant avec lequel il a suivi ces travaux. J'adresse aussi mes remerciements au Gouvernement de la Province de Québec, Canada, qui m'a accordé une bourse d'étude pour recherches scientifiques. 